Before the surgery where did you get information on the operation and living with an artificial eye?

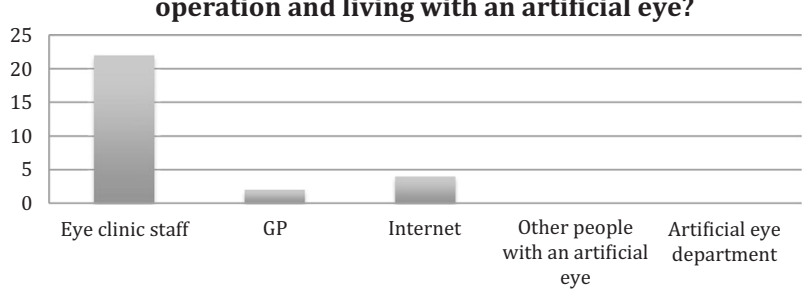

Did you feel that the emotional side of the situation was adequately addressed for your needs?

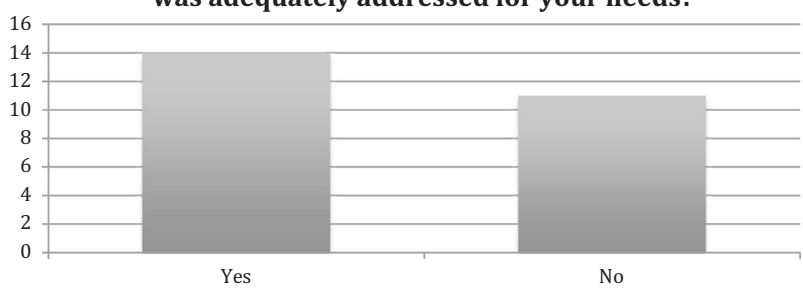

Fig. 2 Graphs showing confidence loss post surgery and adequately addressed emotional needs

limited by emotional problems and mental health disorders [4]. As mental illness remains the leading cause of years lived with disability worldwide [5] and the socioeconomic impact of depression on the UK alone has been estimated annually at over $£ 7$ billion [6], it is vital that we consider the emotional and psychosocial needs of our patients.

\section{Conclusion}

Loss of an eye and the use of artificial eyes have wide ranging emotional and psychosocial impact on patients. Care should not stop when the patient leaves the operating theatre. To maximise postoperative quality of life, a holistic approach, involving counsellors and psychotherapy is essential.

\section{Compliance with ethical standards}

Conflict of interest The authors declare that they have no conflict of interest.

Publisher's note: Springer Nature remains neutral with regard to jurisdictional claims in published maps and institutional affiliations.

\section{References}

1. Abdulkabir AA. Emotional, psychosocial and economic aspects of anophthalmos and artificial eye use. Int $\mathrm{J}$ Ophthal Vis Sci. 2008;7:1-7.

2. Ahn Jm LS, Yoon JS. Health-related quality of life and emotional status of anophthalmic patients in Korea. Am J Ophthalmol. 2010;149:1005-11.

3. Fishbain DGM, Meagher R, et al. Male and femal chronic pain patients categorized by DSM-II psychiatric diagnostic criteria. Pain. 1986;26:18-197.

4. Rasmussen ML. The eye amputated-consequences of eye amputation with emphasis on clinical aspects, phantom eye syndrome and quality of life. Acta Ophthalmol. 2010;88:1-26.

5. Whiteford HA, Degenhardt L, Rehm J, Baxter AJ, Ferrari AJ, Erskine HE. Global burden of disease attributable to mental and substance use disorders: findings from the Global Burden of Disease Study 2010. Lancet. 2013;382:1575-86.

6. Ardino VKM. Counselling and psychotherapy: is there an economic case for psychological interventions? London, UK: British Association for Counselling and Psychotherapy; 2013.

\title{
Raised intra-ocular pressure in the setting of Coffin-Siris syndrome
}

\author{
Michael James Dolaghan ${ }^{1} \cdot$ Sonia George ${ }^{1} \cdot$ Eibhlin McLoone $^{1}$
}

Received: 25 February 2019 / Accepted: 4 March 2019 / Published online: 26 March 2019

(c) The Royal College of Ophthalmologists 2019

Michael James Dolaghan mdolaghan01@qub.ac.uk

1 Department of Ophthalmology, Royal Victoria Hospital, Belfast Heath and Social Care Trust, Antrim, UK
Coffin-Siris syndrome (CSS) is a rare heterogenous genetic disorder first described in 1970. The diagnosis is considered in children with cognitive/developmental delay, 5th finger hypoplasia and characteristic facial features. There is, however, significant variability in the phenotypic appearance, making clinical diagnosis challenging $[1,2]$. 

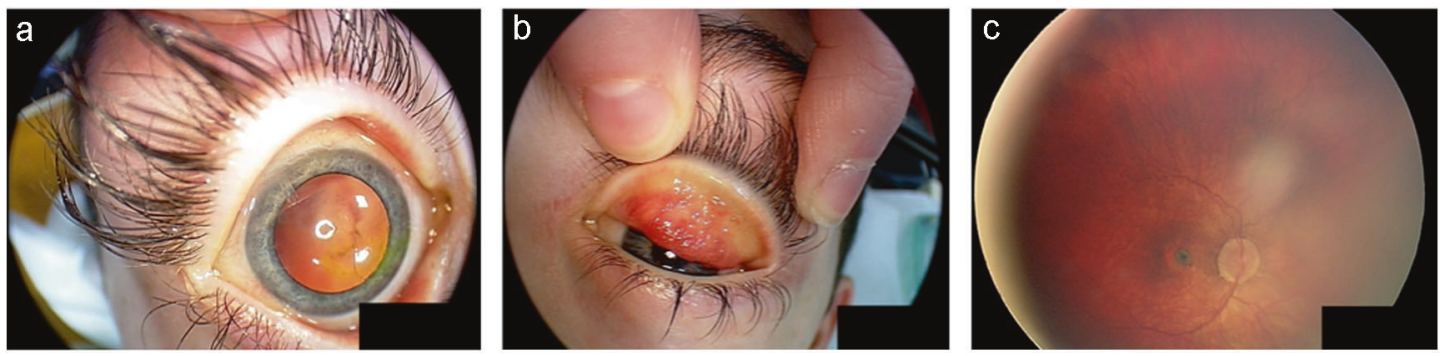

Fig. 1 a Ahmed Valve in situ, b Severe VKC, c Foster-Fuchs spot

Numerous ocular features associated with the syndrome have been reported in the literature: strabismus, nystagmus, cataract, hypophoria, astigmatism, hypermetropia and anisomyopia [3].

We present two cases of children in Northern Ireland with a confirmed genetic diagnosis of CSS (mutation in the ARID1B gene), both having developed significant issues with raised intra-ocular pressure (IOP). We believe glaucoma to be a novel feature of this syndrome.

Our first case exhibited global developmental delay and learning difficulties, hypertonia, small ASD, short stature, hearing loss and speech dyspraxia. The child was found to have a mutation in the ARID1B gene and diagnosed with CSS at 9 years of age. From an ophthalmic perspective, significant anisomyopia was a feature, with the left eye (LE) being more myopic than the right eye (RE), along with a right divergent squint and amblyopia. Severe vernal kerato-conjunctivitis $(\mathrm{VKC})$ presented at age 7 with the development of recurrent shield ulcers. Interestingly, this is not a known association of CSS. In addition to antihistamines and topical Cyclosporin, he was treated with topical and subtarsal steroids for many years. At age 8, he suddenly developed IOPs of $40 \mathrm{mmHg}$ in both eyes associated with optic disc cupping ( 0.4 cup:disc RE and 0.6 cup:disc LE). Despite maximal topical therapy and discontinuing steroids, the child's IOPs remained elevated. Filtration surgery with Ahmed valve insertion was ultimately required on both eyes. VKC was controlled with Omalizumab and all steroids were avoided. Thereafter, IOPs remained stable for 4 years. During this time, he also developed a right Foster-Fuchs choroidal neovascular membrane requiring intravitreal Lucentis therapy. Unfortunately, 5 months ago his pressures suddenly spiked again to a high (30 s) in both eyes, despite having not been on steroids for many years. The role of steroids in the development of his juvenile onset glaucoma remains unclear (Fig. 1).

Our second case was referred to ophthalmology at age 13 weeks following an antenatal finding of Corpus Callosum agenesis. After genetic testing she was diagnosed with CSS, possessing the same mutation as our first case. The patient exhibited developmental delay and delayed visual

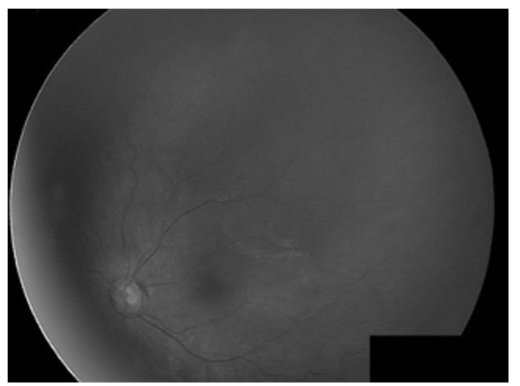

Fig. 2 Glaucomatous optic disc cupping

maturation, with progressive anisomyopia being the most significant ocular feature associated with right divergent squint and amblyopia. From 4 months, rising IOPs were noted in both eyes, associated with disc cupping. Interestingly, the least myopic eye developed the more advanced glaucomatous disc cupping. Currently her pressures are managed with topical therapy (Fig. 2).

Both our cases display already reported ocular features of CSS—progressive anisomyopia and unilateral divergent squint with associated amblyopia. However, the development of glaucomatous features common to both cases is interesting and not previously reported in the literature. The exact mechanism of this open-angle glaucoma is unclear, and further investigation into the structural ocular abnormalities present in CSS is necessary to evaluate whether this genotype (ARID1B mutation) is linked to the development of glaucoma.

\section{Disclaimer}

The authors declare that the material presented is original research, has not been previously published and has not been submitted for publication elsewhere while under consideration.

\section{Compliance with ethical standards}

Conflict of interest The authors declare that they have no conflict of interest.

Publisher's note: Springer Nature remains neutral with regard to jurisdictional claims in published maps and institutional affiliations. 


\section{References}

1. Schrier SA, Bodurtha JN, Burton B, Chudley AE, Chiong MAD, D'avanzo MG, et al. The Coffin-Siris syndrome: a proposed diagnostic approach and assessment of 15 overlapping cases. Am J Med Genet A. 2012;158A:1865-76.
2. Levy P, Baraitser M. Syndrome of the month: Coffin-Siris syndrome. J Med Genet. 1991;28:338-41.

3. Pallotta R. Ocular anomalies in Coffin-Siris syndrome. Ophthalmic Paediatr Genet. 1985;6:109-12.

\title{
Digital photo-editing in preoperative counselling for cosmetic corneal tattooing
}

\author{
Tariq Ayoub $^{1} \cdot$ Tom Henry Flynn ${ }^{1}$
}

Received: 6 March 2019 / Accepted: 18 March 2019 / Published online: 28 March 2019

(c) The Royal College of Ophthalmologists 2019

Corneal tattooing is a well-described procedure for reconstructive cosmetic purposes [1] and for optical purposes [2]. It can serve as an excellent alternative to the use of cosmetic contact lens. However, it is often difficult to explain to patients the cosmetic outcome of the procedure. We describe the use of digital image editing software to aid preop counselling.

\section{Case}

A 42-year-old man presented with a blind left eye and a scarred and opaque left cornea after childhood trauma. He was bothered by the appearance of the eye (Fig. 1a). He failed a trial of cosmetic contact lens-he found it inconvenient and hard to handle the lens and eventually lost it. He was offered corneal tattooing but was concerned with the post-operative cosmetic outcome of the procedure.

To aid preoperative counselling, we used digital photograph-editing software (Powerpoint, Microsoft, Redmond, WA, USA) to alter a picture of his face and simulate the outcome of corneal tattooing (Fig. 1b). After counselling, he was keen to proceed.

Tom Henry Flynn

tom.flynn@nhs.net

1 Moorfields Eye Hospital, 162 City Road, London EC1V 2PD, UK
The surgery was carried out using black sterile medical ink used in radiotherapy patients (off-label use). A 4-mm "pupil" was marked on the corneal surface with a skin trephine dipped in gentian violet. A 300-micron incision was made circumferentially around this mark for $90^{\circ}$. A lamellar dissection was performed within the limits of the "pupil" mark. The ink was injected and spread within this pocket and the incision was closed with a single interrupted 10-0 nylon suture. The tip of a curved needle from a 10.0 nylon suture was dipped in the ink and used to mark the peripheral cornea with a series of short tangential passes in order to simulate the slightly darker appearance near the limbus of light irides. The patient was prescribed dexamethasone $0.1 \%$ qds and $\mathrm{G}$ Chloramphenicol $0.5 \%$ qds post operatively.

post-operative appearance closely resembled the preoperativesimulation and the patient was pleased (Fig. 1c).

\section{Discussion}

Failure to meet the cosmetic expectation and perceived deficits in informed consent can cause dissatisfaction for patients after facial cosmetic procedures [3]. We report a case of a satisfied patient where the outcome of surgery was very similar to that seen in the simulation created preoperatively on photo-editing software. Use of such software is an easily accessible and effective tool to counsel patients of the outcome of cosmetic corneal tattooing preoperatively. 